\title{
Celiac Disease and Its Association with Organ- Specific Auto-Immune Diseases
}

\author{
Sakshi Buchke ${ }^{1}$, Anusuiya Bora ${ }^{2}$, Bhavika Mehta $^{2}$, Maitrali Relekar ${ }^{3}$, Piyush Varsha ${ }^{4}$, \\ Jitendra Kumar ${ }^{5}$, Piyush Bhanu ${ }^{* 1}$ \\ ${ }^{1}$ Xome Life Sciences, Bangalore Bioinnovation Centre, Helix Biotech Park, Bengaluru, Karnataka \\ ${ }^{2}$ School of Bio Sciences and Technology, VIT, Vellore Campus, Tiruvalam Rd, Katpadi, Vellore, Tamil Nadu \\ ${ }^{3}$ KEM Hospital Research Centre, KEM Hospital, Rasta Peth, Pune, Maharashtra \\ ${ }^{4}$ Saraswati Dental College, 233, Faizabad Rd, Tiwari Ganj, Chinhat, Lucknow, Uttar Pradesh \\ ${ }^{5}$ Bangalore Bioinnovation Centre (BBC), Helix Biotech Park, Electronics City Phase 1, Bengaluru, Karnataka
}

*Corresponding Author: Piyush Bhanu; pb.inresearch@gmail.com

Received 04 September 2021;

Accepted 25 September 2021;

Published 01 October 2021

\begin{abstract}
Wheat is one of the most consumed foods in the world. Although it is extremely nutrient rich for us humans, some of us have great difficulties in completely digesting its protein subunits. This review aims to understand the onset of Celiac Disease and its association with several other autoimmune diseases. The gliadin molecule, undigested in the small intestine, over time, ruptures the villi lining of the intestinal wall and enters the bloodstream which in turn activates the body's immune response. In some patients with the presence of HLA DQ2/DQ8 genes, this immune response results in Celiac Disease. Notably, researchers over the past several decades have found several links between Celiac Disease and multiple auto-immune diseases. Diabetes is one such auto-immune disease which has shown multiple associations with Celiac Disease. Similarly, in this review paper, we are critically analyzing the association of Celiac Disease with some of the most common autoimmune diseases namely Type-1 Diabetes, Multiple Sclerosis, Autism and Inflammatory Bowel Disease. In this paper, we have shown a clear correlation of celiac disease with several other auto-immune diseases. Further study is needed to understand the bidirectional association of Celiac Disease with different auto-immune diseases.
\end{abstract}

Keywords: Celiac disease, Type 1 diabetes, Multiple Sclerosis, Autism Spectrum Disorder, Inflammatory Bowel Disease.

\section{Introduction}

Wheat, a widely cultivated cereal grain, is one of the most consumed staple foods in the world. Wheat (white and whole wheat) is the major ingredient that is used in baked goods such as sliced breads, pasta, noodles to name a few. The whole-grain wheat is a rich source of fibers, vitamins, minerals and antioxidants (Awika, 2011).

The chemical composition of wheat includes $80 \%$ of carbohydrates out of which $70 \%$ is starch and nearly $5 \%$ of polysaccharides, $12 \%$ of proteins and $2 \%$ of lipids (Knudsen \& Hansen, 1991). The major protein component found in wheat is gluten. It comprises $80 \%$ of all the protein that is present in wheat (Shewry et al., 2002). Gliadin and glutenin are the two major components of gluten and they are responsible for determining the functional properties of wheat. Gluten has adhesive properties that helps the food to maintain its form and shape, provides elasticity and provides the chewy structure in the food. This was made possible through the cross-breeding process allowing the wheat to have more gluten components and thus improving the overall stability of food (Wrigley et al., 2006).

Gliadin is a type of protein and the major sub-component $(53 \%)$ of gluten that is present in wheat. The intramolecular disulfide bonds make the compound water-insoluble. Based on its amino acid composition, gliadin is further divided into three major types namely, alpha/beta, gamma and omega. The amino acid composition contains repetitive sequences of hepta or dodeca peptides of proline and glutamine. These prolamine are resistant to human digestive enzymes. Since gliadin is water-insoluble, it is extremely difficult for humans to digest the protein (GarciaBennett et al., 2016; Shwery, 2019). The major cause of concern for humans is that gliadin is an insoluble molecule and its consumption over time can slowly rupture the intestinal lining after which, the gliadin molecule can cross the intestinal epithelial lining and go into the blood vessels which can further cause celiac disease (Fasano et al., 2011). Some research papers have also explored the possibility that the breast milk of healthy human 
mothers who have consumed the gluten-based foods, have shown the presence of non-degraded gliadin. The presence of these gliadin antigens in the mother's milk is of importance because they could be involved in the modulation of the immune response in neonates (Chirdo et al., 1998).

Celiac disease (CD) is an auto-immune disorder and primarily affects the small intestine. Although it affects the intestinal walls, it is not just a gastrointestinal disease but a multi organ chronic problem. However, CD affects almost $2 \%$ of the world population but the problem remains unrecognized and undiagnosed (Caio et al., 2019). CD has also been associated with several other auto-immune diseases such as type-1 diabetes, autism, thyroiditis (inflammation of the thyroid gland), psoriasis (non-contagious skin absorbability), autoimmune hepatitis and others (Lundin \& Wijmenga, 2015). Some of the most commonly occurring auto-immune diseases were chosen for this review. In this paper, we are doing an in-depth review on celiac disease and exploring associations with several auto-immune diseases that have been associated with $\mathrm{CD}$.

\section{Celiac Disease}

Celiac disease (CD) is a gluten-sensitive enteropathy (GSE) and an immune-mediated condition. It is a disease that occurs extensively in the small intestine (Mocan \& Dumitraşcu, 2016). Patients with CD tend to develop small bowel villous atrophy, malabsorption, and weight loss (Willoughby, 2014). All of this can be avoided by implementing a strict gluten-free diet (GFD) in one's lifestyle.
Clinical CD characteristics vary by age group. Infants and toddlers may have diarrhea and abdominal distention; older children may have short stature, neurological symptoms, and anemia; adults may have diarrhea and abdominal pain classically, but may have extra-intestinal characteristics, such as anemia with iron deficiency and premature bone metabolic disease (Kamboj \& Oxentenko, 2017).

The main suspect for the development of celiac disease seems to be Gliadin (De Re et al., 2013). Gliadin is a peptide found in food products containing gluten, which induces inflammation when consumed due to activation of helper T-cells (Pilli et al., 2017). Nutrient malabsorption is characterized by inflammation due to damage to the villi of the small intestinal mucosal tissue.

The initial $\mathrm{CD}$ screening test is an anti-tissue transglutaminase (tTG) immunoglobulin A ( $\operatorname{Ig} \mathrm{A}$ ) antibody, with the exception of patients with confirmed or suspected $\operatorname{IgA}$ deficiency in which IgG-based serology is required (Kamboj \& Oxentenko, 2017). A significant development was the discovery of the tTG IgA antibody as the target antigen for IgA antibodies. tTGbased studies also placed CD-specific serology into the scope of being used for diagnosis by most physicians and hospitals (RubioTapia et al., 2013).

Enzymes that are responsible for degrading intact proteins into single amino acids, di-peptides and tripeptides are secreted from the liver, walls of the small intestine, and pancreas during the digestion phase. Gluten is transformed by tissue transglutaminase (tTG) into its components, gliadin, and glutenin, during intestinal proteolysis, as protein-rich products that enter the intestine (Aram et al., 2015).

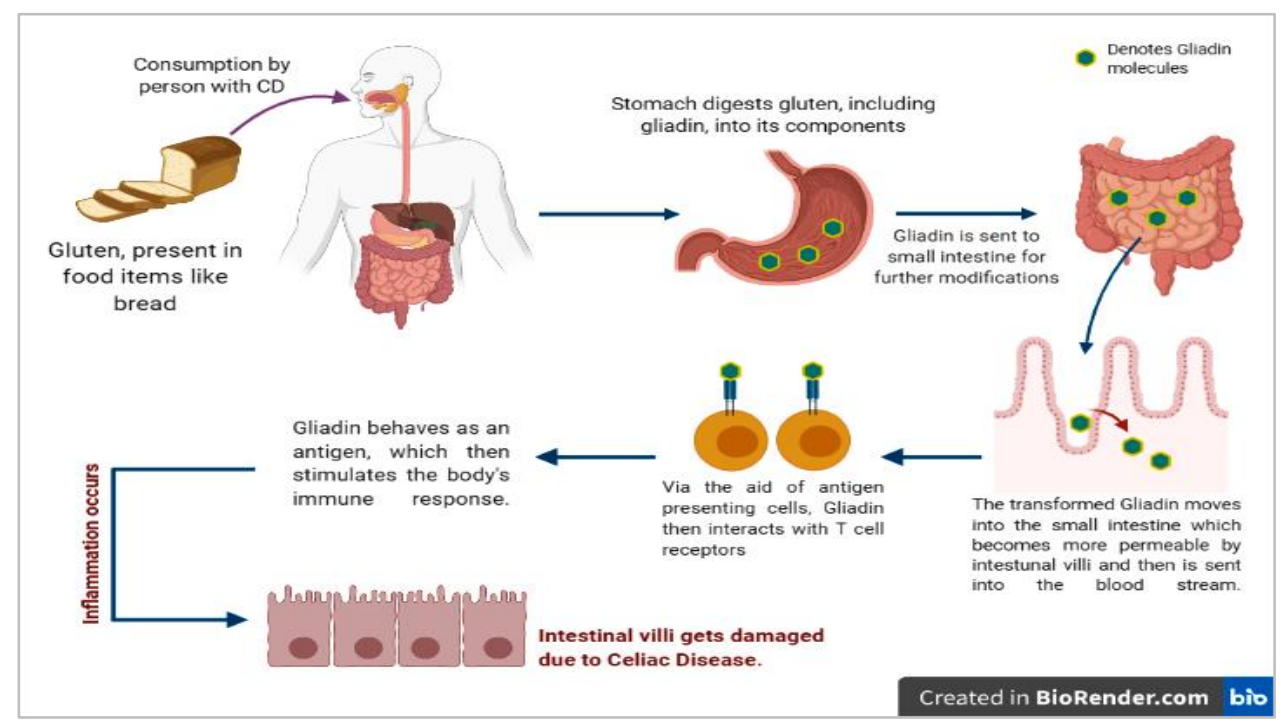

Figure 1: Gluten consumption and the onset of celiac disease. The flowchart demonstrates the mechanism of intake of gluten-containing food products and then the absorption of gliadin, which results in the effect of celiac disease on small intestine villi.

Due to its smaller molecular mass, which gives it a wide surface area for the enzymes to bind, Glutenin is readily degraded by the digestive enzymes, resulting in easy degradation. However, due to the fact Gliadin is tightly packaged, it is resistant to enzymatic degradation as it has low surface area to volume ratio for enzyme action to take place (Stepniak et al., 2006). Gliadin is digested into oligopeptides instead of di or tri-peptides (Aram et al., 2015). Thus, gliadin passes through the intestinal leaky junctions and meets tissue transglutaminase (tTG) in the intestinal lumen. The resulting macromolecular complex is presented to the antigen presenting cells. The adaptive immune system is activated when the $\mathrm{T}$ cells recognize and bind to the gliadin epitope to form CD4 cells thereby releasing the pro inflammatory cytokines. These cytokines induce the degradation of the intestinal mucosal matrix and cause cell death. Gluten-specific antibodies and transglutaminase specific antibodies are produced by B cells. Thus, the resulting effect of these gluten digestion-formed oligo-peptides is the mediation of an inflammatory reaction during the digestion of gluten. When antibodies target gliadin and tTG, this ultimately results in an autoimmune reaction which then adversely affects the digestion of gliadin.

Research in recent past has established that increased intestinal permeability in MS has been observed due to the involvement of gliadin. In the gut, lesions can also be caused by celiac disease. This would increase the intestinal permeability, resulting in higher absorption of gluten and gliadin (Shimada et al., 
2019). Hence, this results in an increase in IgA antibodies against gliadin protein present in blood samples.

With reference to Multiple Sclerosis, most studies have found the prevalence of antigliadin antibodies in MS patients by tests conducted and findings provided on MS patients. On the other hand, $\mathrm{CD}$ exhibits neurological dysfunctions including ataxia, neuropathy of the periphery, and seizures. In celiac patients, multiple sclerosis-like diseases, and headaches associated with brain MRI white matter lesions have also been identified (BaturCaglayan et al., 2013).

Gluten ingestion is dependent on the antibodies directed against gliadin or its deamidated products as well as the selfantigen tTG (Rubio-Tapia et al., 2013). Reduction or elimination of dietary gluten contributes to a natural reduction in amounts of the levels of all the celiac-associated antibodies. As a preventive measure, a gluten and consequently, gliadin free diet by eliminating the intake of wheat, barley and rye, is advised to the patients of Celiac disease (Kamboj \& Oxentenko, 2017). Therefore, it is important to get screened against compounds and ingredients that are usually present in our diet.

\section{Celiac Disease and Human Pancreas}

\section{Association of Celiac Disease (CD) with Diabetes Mellitus Type 1 (T1D):}

Type 1 Diabetes or Insulin-Dependent Diabetes Mellitus (IDDM) is a chronic metabolic disease arising due to deficient production of insulin, which is often because the pancreatic beta-cells are destroyed as a result of autoimmune response. It is a multifactorial disease, a certain disharmony between predetermined high risk genetic makeup accompanied with triggering external environments, which include food, viral encounters, stressful events, changes in gut microbiota and others that lead to onset of T1D. In T1D patients, gut microbiome includes bacteria from phylum Bacteroidetes, and reduced number of Bifidobacterium, while healthy individuals have abundant Firmicutes (Giongo et al., 2010; de Goffau et al., 2012; Murri et al., 2013). Introduction of cereals from age of 3 months to 7 months keeps genetically susceptible infants safe from T1D and CD, and continuation of breastfeeding along with cereals show a protective effect in these infants (Norris, 2003; Ziegler, 2003; Norris, 2005; Virtanen \& Knip, 2003; Akonbeng, 2005). Enteroviruses pose an increased threat for these infants as well (Yeung et al., 2011; Stene et al., 2010).

Generally, the onset occurs after a long latent preclinical phase during the course of which autoimmune responses are generated in the cells, which stress and later destruct the the beta cell mass of pancreas, which in turn results in lower secretion of insulin thereby resulting into high glucose concentration in the blood (hyperglycemia).

Genetic approach to T1D states that particular HLA haplotypes like DQ2 and DQ8, or their trans-dimers (DQ2/DQ8) show relevance in activation of autoimmune T-cells in patients (Quan et al., 2019; Ludvigsson et al., 2013). HLA-DR molecules help in presentation of antigenic peptides on the cell surface (Calderoni et al. 2016), and the selection of the high-stability antigenic peptide is driven by HLA-DM (Pociot \& McDermott, 2002). Interruptions in edit of DM can result in presentation of wrong peptide, inducing the autoimmune T-cells, which might result in pathogenesis (Zhou et al., 2016). A structural study shows rearrangements in the complex of HLA-DR and HLA-DM which often lead to removal of peptide present in the peptide-binding groove, for a high stability antigenic peptide to bind (Calderoni et al., 2016). Affinity of DQ2 for DM is naturally very substandard and this serotype is often found in T1D patients (Pos et al., 2012). Reason for HLA-DQ8 involved in development of T1D is not fully explored and understood.

As the fairly resistant gliadin fragments cross the intestinal barrier, they accumulate in the intestinal region. Beta cells, after exposure to gliadin, secrete insulin in both stimulated as well as resting conditions. This creates stress in the cells along with local inflammation caused due to proinflammatory cytokines. Several other immune responses are generated, which create an imbalance in the system. Gliadin plays a role in hyperactivity of beta-cells prior to the onset of T1D (Jensen, 2007).

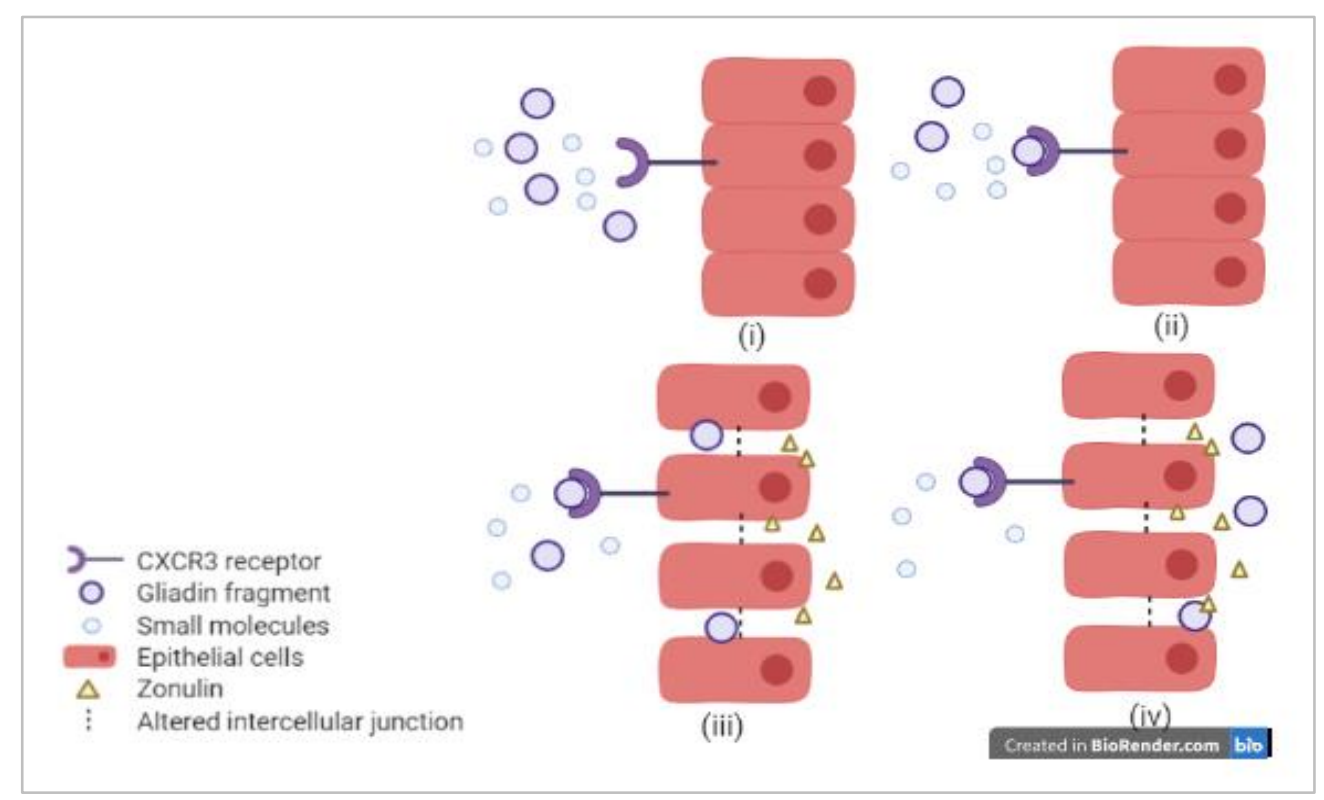

Figure 2: Receptor mediated cellular uptake. Receptor mediated cellular uptake is a fairly regulated process, but when the tight junctions lose their integrity and the gap widens intercellularly, the gliadin fragments enter the cell at a much higher rate, causing hyperactivity of beta-cells for insulin production, and thus the beta cells are stressed. The inflammation results in a cascade of other immune reactions and thus production of autoantibodies, which might trigger the onset of T1D. (i) Gliadin fragments are found in the bloodstream (ii) Gliadin fragment binds on the receptor for cellular uptake (iii) the iTJ widens and exhibit an altered function due to production of Zonulin (iv) Excess gliadin fragments enter into the pancreas. 
In a subgroup of patients suffering from T1D, Zonulin, a protein that maintains the permeability of intestine, breaks apart the tight junctions present intercellularly. Zonulin is often associated in innate immune responses.

A study by Sapone et al. (2006) in Bio breeding Diabetes-Prone (BBDP) rats showed that due to its upregulation, the intestinal permeability increases in T1D patients. This acts as a connection between enhanced permeability of intestine and exposure to foreign antigen during the onset of the disease in individuals who are susceptible genetically (Yang et al., 2014). Watts et al. (2005) in their study showed that use of Zonulin inhibitors actually block the formation of autoantibodies and reduce diabetes incidences in a particular subgroup. There was a significant pattern, where incidences of upregulation of Zonulin and decrease in measurement of transepithelial electrical resistance (TEER) was noted in Diabetes-Prone BB (BBDP) rats (Fallang et $a l ., 2008)$. The measurement of transepithelial electrical resistance helps to determine the integrity of tight junctions (Dall et al., 2013). This decreased TEER was followed by autoantibody synthesis against the beta cells, which is then followed by the clinical onset of T1D (Sapone, 2006).

Bosi et al. (2006) in their study on bio-breeding rats demonstrated alterations in intestinal permeability to sugars via lactulose-mannitol test. Inside the intestinal mucosa, mannitol moves via a transcellular pathway whereas large lactulose is transported paracellularly. But with altered integrity, as in case of T1D, an unusual uptake of lactulose is noted, which indicates damaged barrier function of the mucosa. High uptake results in the presence of excess lactulose in urine, as in the T1D patients (Watts et al., 2005). Brun et al. (2016) conducted a study in NOD mice where they fed them with 33-mer and 19-mer labelled gliadin peptides resistant to enzymatic proteolysis. After administration, a large number of radioactive labels could be witnessed in the exocrine region of the pancreas, stating an altered barrier function that allowed these peptides inside the pancreas (Srinivasan et al., 2015).

The source of dietary protein is important for the onset of T1D. Scott and his co-workers (1991) designed different diets for diabetes prone BB rats with similar nutritional values but had significant differences in protein sources, with a casein-based control diet. It was concluded that wheat gluten had a peculiar structure which could be associated with more incidences of diabetes than any others fed to the group of rats. Cereal diets appear to be more diabetogenic than semi-purified diets based on casein in hydrolyzed or regular forms (Bosi et al., 2006).

A gluten-free diet (GFD) has been under focus by several researchers. When NOD mice are fed on a GFD diet by Funda et al. (1999), the incidences of diabetes are around $15 \%$ whereas mice fed with a standard diet had $64 \%$ incidences of diabetes within around 320 days of the study. GFD significantly delayed the onset of diabetes, and for mice who were never fed gluten, it prevented the occurrence of diabetes (Brunn et al., 2016). Marietta et al. (2013) made a significant observation on different composition of fecal microbiota in NOD mice who were fed GFD as compared to mice fed a gluten-diet. The former also led to decreased incidences of hyperglycemia in NOD mice, but it was reversed as soon as diet was reintroduced with gluten (Marietta et al., 2013).

In another study by Pastore et al. (2003), when first degree relatives of diabetic patients who possessed 2 or more autoantibodies, namely insulin autoantibodies, GADA and IA-2A were given GFD diets for a span of 6 months, the insulin resistance decreased and the sensitivity increased. But, once they were restored to normal diets, the insulin sensitivity decreased again.
This study concluded that GFD diet has a protective role for functioning of beta-cells in individuals susceptible to development of T1D (Scott \& Marliss, 1991). GFD diet had a positive impact on insulin secretion. A study was conducted in kids of mean age as 7.5 years who were susceptible to T1D by Saadah et al. (2004), which showed that they had antigliadin IgG and IgA antibodies. With GFD diet, the weight and BMI of these kids increased over a period of a year, and their insulin production also increased (Funda et al., 1999).

The DR3-DQ2 genotype is strongly associated with celiac disease (CD) (Bao et al., 1999), along with its relevance in T1D. It also appears that autoantibodies related to diabetes are found in patients suffering from CD (Galli-Tsinopoulou et al., 1999). Antibodies against tissue transglutaminase or tTG have been detected in NOD mice, which supports the hypotheses of close links of these two disorders (Santiago et al., 2008). Also, polymorphism in Myosin IXB can be involved in alterations in permeability of intestine and thus the development of CD and T1D (Sblattero et al., 2005).

Among children suffering from T1D, prevalence of $\mathrm{CD}$ ranged from $0.97-16.4 \%$ in 26 different screening reports (Holmes, 2002). Another report by Volta et al. suggests prevalence to be roughly $8 \%$. CD at the onset of T1D is less, almost $1 \%$ but it can occur after several years (10 years) of diagnosis of T1D. This makes serological very essential in the following years (Volta et al., 2011).

A study by Pocecco et al. suggests that in the majority of the patients, T1D is diagnosed before CD (Pocecco \& Ventura, 1995). CD patients have several gastrointestinal and extraintestinal troubles, but contrastingly reported by Valerio in T1D patients, the presentation of gastrointestinal symptoms is either rarely mild or just completely absent (silent CD) (Valerio, 2002) short stature, anemia due to iron-deficiency, reduced BMI, vitamin K deficiency and excess bleeding are some systemic signs in T1D patients suffering from CD (Poulain et al., 2007). Study by Ventura et al. indicates the prevalence of T1D and other autoimmune disorders can be prevented to an extent with early diagnosis of $\mathrm{CD}$. This would help reduce the exposure of the intestine to gluten and thus reducing the chances (Ventura et al., 2000).

Osteopenia seems to be a hidden threat for individuals suffering from T1D and CD simultaneously, rather than just one of the two. It can also be connected with dietary compliance to GFD (Camarca et al., 2012). Untreated CD patients have imbalanced cytokines which lead to compromised bone metabolism, which affects its activity (Lombardi et al., 2010).

\section{Celiac Disease and Human Brain}

\section{Association of Celiac Disease (CD) with Multiple Sclerosis (MS):}

Multiple sclerosis (MS) is characterized as a deteriorating disorder that affects the central nervous system (CNS). The immune system begins destroying the sheath of myelin that covers the nerve fibers and protects them. The intervention results in nervous system inflammation. Myelin enables the nerves in the body to effectively conduct electrical signals. Therefore, it is an integral part of the nervous system. Thus, in areas of the CNS, MS results in gliosis and demyelination accumulation.

A T cell-mediated inflammatory disease of the CNS is commonly believed to be MS. There have been studies in which inflammation mediated by B cells of the immune system is often held responsible for the disease's progression. MS affects 
approximately 1 percent of the world's population, primarily young women (San Mauro et al., 2016).

There are different forms of MS that can occur in one's body. In this review, we will discuss the relationship between Gliadin, a type of protein that is a gluten component present in wheat, and Multiple Sclerosis in general.

Even though, so far, no direct relation has been identified between MS and Gliadin. A research study was carried out by K.L. D. and Reichelt. Jensen, about patients who volunteered with the Norwegian Multiple Sclerosis Society. With an age range of 27-67 years, the total number of MS patients was 36 (median 44 years). There were 21 females and 15 males out of them. Normal controls of 26 individuals were collected by the Fursts laboratory in Oslo (high quality Clinical Chemistry Laboratory) who were 21-50 years of age (median of 38 years). 16 of them were females and 8 of them were males (Reichelt et al., 2004).

Venous blood was collected and the ELISA technique tested the IgA and IgG antibodies. The following statistics were obtained after the test:

The sample containing gluten against the control showed serum IgA antibodies that were higher than controls with (statistical P test values) $p<0.001$ (two-tailed). IgA antibodies against gliadin also had $p<0.001$. This indicates a good level of significance of the presence of antibodies in the body due to the presence of gluten and consequently, gliadin. The IgG antibody levels against gluten were different from controls with $p<0.001$. Subsequently, for gliadin a $p<0.001$ was obtained (Reichelt et al., 2004).

As per the study that was conducted, a highly significant difference from the controls was observed in serum IgA antibodies against gliadin and gluten which was extremely significant $(p<$ 0.001) (Reichelt et al., 2004). An increase in specific serum IgA antibodies is thought to reflect the increased uptake of protein from the gut and its lining. This could tend to more gluten and hence, gliadin being absorbed by the body, causing other diseases like Celiac Disease. CNS demyelination has also been linked with other gastrointestinal illnesses where the intestinal barrier is compromised (Camara-Lemarroy et al., 2018). As a result, MS patients have been reported to tend to have more anti-gliadin antibodies in their immune system.

Both Multiple Sclerosis (MS) and Celiac Disease (CD) are considered to be T-cell-mediated autoimmune diseases, and the involvement of Th1 cells in the pathogenesis of $\mathrm{CD}$ has been reported (Jabri \& Sollid, 2017; Zhang et al., 2020).

There are few reports from patients who had an indication of the prevalence of MS and gluten sensitivity. The brain and spinal-cord white matter lesions on MRI reports of patients have been an indicative factor to diagnose MS in patients who have been experiencing problems related to $\mathrm{CD}$.

From a case report of a patient of $\mathrm{CD}$, it was established that the patient had anti-gliadin antibody of isotype $\operatorname{IgA}$ along with other biomarkers in her serum sample [66]. Initially, her records had indicated chronic gastritis associated with Heliobacter pylori and chronic duodenitis. This was not an indicative measure of celiac disease, however, when the patient was subjected to a gluten-free diet (GFD), her GI symptoms had subsided. Hence, the diagnosis of celiac disease was done. As the years passed by, the patient had episodes of various neurological disorders like ophthalmoplegic migraines, diplopia that gives a perception of two images of an object at a certain axis. Tests done for the brain MRI indicated a demyelinating condition of plaques without any structural abnormality in the cerebellum. Various other neurological factors led to the clinical diagnosis of MS (Shaygannejad et al., 2013).

Another case report of a patient was studied who was initially diagnosed with MS and slight symptoms of CD. She was admitted to the hospital with hemi-hypoesthesia (partial loss of sensation in parts of the body). Test reports also indicated iron deficiency and the prevalence of irritable bowel syndrome (BaturCaglayan et al., 2013). Brain MRI hyperintense abnormalities in the white matter region. The cerebrospinal fluid investigation represents a mildly increased $\operatorname{IgG}$ index. The patient was treated with the medication of intravenous methylprednisolone for 5 days which showed improvement in motor and sensory symptoms. gradually improved. After a span of a year, she was readmitted to the hospital three times with symptoms like right hemiparesis, visual blurring in the right eye, dizziness, and diplopia. She was given the drug - methylprednisolone for ten days. Reports of the brain MRI indicated definitive signs that led to the diagnosis of MS. Even though the neurological conditions were being stabilized, the matter of concern was the persistent deterioration of iron levels in her body. This led to celiac screening for the antibodies: antigliadin (AGA) IgA and tissue transglutaminase antibody (TTG). Test results were positive. Gastrointestinal endoscopy was conducted that indicated antral gastritis and duodenopathy. Through biopsy, blunting of villi was also reported that indicated the onset of celiac disease. The patient was hence, given a gluten-free diet for the management of celiac disease (Batur-Caglayan et al., 2013).

Research over the years has established that approximately $10 \%$ of those who have MS are also susceptible to celiac disease (Rodrigo et al., 2011). From the case studies that have been reported so far, MS has been linked with $\mathrm{CD}$ as a progressive disorder that may take place in due course of time after the onset of Celiac Disease. One should take precautionary measures and get a diagnosis of food tolerance done to avoid further complications.

\section{Association of Celiac Disease (CD) with Autism Spectrum Disorder (ASD):}

Autism as well as Autism Spectrum Disorders (ASDs) are neurodevelopmental disorders (Lahiri et al., 2013). The characteristics of Autism include impaired social interactions and communication, restricted and repetitive behavior, interests and activities (Faras et al., 2010). Gastrointestinal (GI) problems are common in the individuals suffering from ASD (Chaidez et al., 2014). Studies show that children with ASD have around four times more gastrointestinal symptoms than the unaffected children (McElhanon et al., 2014). Usually, the food intolerance in ASD patients goes unrecognized because of their inability to express their discomfort (Cermak et al., 2010).

The increased permeability of the gut and the blood brain barrier might play a role in this (Fiorentino et al., 2016). The autonomic nervous system has been divided into three parts, sympathetic, parasympathetic and enteric (Waxenbaum et al., 2020). The gut has its own nervous system which is known as the enteric nervous system (ENS) consisting of millions of neurons (Schneider et al., 2019). The ENS can control the gastrointestinal behavior independently, that is, without any support of the brain and spinal cord. It has been reported that the genetic defects that affect the CNS function might also affect the ENS function in case of ASD (Rao \& Gershon, 2016).

Genetic and environmental factors play a key role in the development of autism (Bai et al., 2019). Vojdani et al., in their earlier study suggested that dietary proteins like gliadin play a role 
in the pathogenesis of autism. These peptides bind to the lymphocyte receptors which triggers the autoimmune reactions in children with autism (Vojdani et al., 2003). Also, in a separate study by Vojdani et al., it has been found that a significant percentage of the sera of autistic patients has elevated levels of IgG, IgM, or IgA antibodies against gliadin (Vojdani, 2004). Elevated levels of these antibodies against gliadin in autistic patients in comparison to the healthy controls were also found in a similar study by El-alameey et al. in 2018 .

The 'leaky gut' hypothesis states that because of the defect in the intestinal tract permeability, various bacteria, toxic metabolites and small molecules pass into the bloodstream (Hollander \& Kaunitz, 2020). Under normal circumstances, gliadin is unable to enter the gut associated lymphoid tissue (GALT) because of the presence of tight junctions along the intestinal tract (Fasano, 2011; Schneeberger \& Lynch, 2004). It has been demonstrated that binding of gliadin to a chemokine receptor CXCR3, leads to the release of Zonulin which results in the subsequent increase of the intestinal permeability (Lammers et al., 2008). Vojdani et al., in another study showed that $33 \%$ of children with ASD tested positive for IgA antibodies against CXCR3binding gliadin peptide (Vojdani \& Vojdani, 2017).

Maternal IgG antibodies are transferred from the mother to the child through the placenta (Fouda et al., 2018). This provides the child with the passive immunity against the antigens (Slifka \& Amanna, 2018). A recent study conducted by Gardner et al., showed that the high level of maternal anti-gliadin antibodies protects the child against ASD (Gardner et al., 2020).

There has been very little evidence suggesting a correlation between CD and ASD. Majority of the studies found no association between the two (Quan et al., 2019). However, there are a few reports which might suggest a correlation. In a Swedish investigation, conducted on around 27,000 CD individuals, a strong association was found between the CD patients with a normal mucosa but having positive CD serological test results and ASD (Ludvigsson et al., 2013). In another study on 382 preschoolers with ASD, the prevalence of $\mathrm{CD}$ was found to be $2.62 \%$ (Calderoni et al., 2016).

It was suggested by Liberto et al., that certain peptides are generated in $\mathrm{CD}$ patients which bind to the opioid receptors after crossing the blood brain barrier and are harmful for the brain functioning. They also suggested an association between the CD and ASD because of increased oxidative stress and mitochondrial dysfunction (Di Liberto et al., 2020). Furthermore, Barcia et al., followed by their investigation recommended that all children with autism should be screened for $\mathrm{CD}$ even if gastrointestinal symptoms are absent (Barcia et al., 2008).

\section{Celiac Disease and Human Intestine}

\section{Association of Celiac Disease (CD) with Inflammatory Bowel Disease (IBD):}

Understanding the association between $\mathrm{CD}$ and autoimmune disease IBD is crucial because it could have an effect on CD clinical supervision and screening methods (Pinto-Sanchez et al., 2020).

Exact cause of IBD is still not known. It is believed that IBD is caused by an abnormal immune response to unknown external factors in genetically compromised individuals (Malik et al., 2015).

When it comes to the association of IBD and CD, there are several genetic, environmental and microbial factors involved. Several case reports have been published detailing the outcomes of patients with celiac disease and IBD (Bulger et al., 1988; Casella et al., 2010; Cottone et al., 2003; Gillberg et al., 1982; Kitis et al., 1980; Kumar et al., 1979; Lawlor \& Peppercorn, 2011; Leeds et al., 2007; Ordonez et al., 2012; Patel et al., 2011; Schedel et al., 2005; Yang et al., 2005). IBD basically comprises of two conditions: Crohn's disease and ulcerative colitis; out of which, Crohn's and CD share genetic risk loci, including PTPN2, IL18RAP, TAGAP, and PUS10 (Festen et al., 2011). Common symptoms include increase in intestinal permeability (Camilleri et al., 2012), irregularity in T-cell function (Hmida et al., 2012; Soukou et al., 2018) and proinflammatory cytokines such as interleukin IL-17, IL-21, and (IL)-15 (Jabri \& Abadie, 2015; Miesel et al., 2017). Additionally, interferon-gamma (IFN- $\gamma$ ) (Hisamatsu et al., 2016) and some microbial factors play their role in both the diseases (Harris et al. 2018; Caminero et al., 2019).

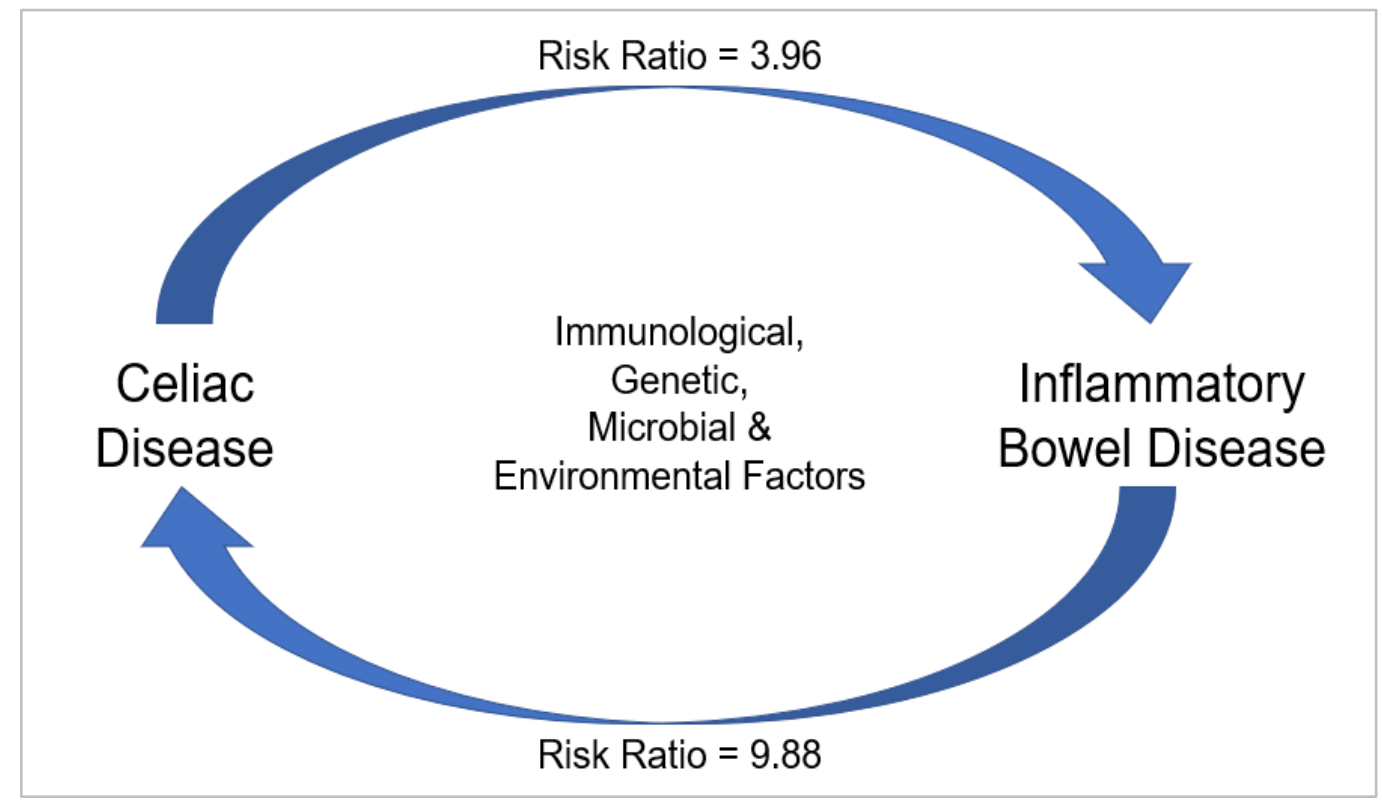

Figure 3: Bidirectional association of IBD and CD. There is nine times increased risk of IBD in CD patients, greater risk of Crohn's disease as compared to ulcerative colitis. Various factors are involved in the prevalence of the diseases as shown in the figure. (RR=Risk Ratio (Pinto-Sanchez et al., 2020) 
CD occurs because of susceptibility presented by HLA-DQ2 or HLA-DQ8 genes as well as gluten and prolamin intake via food (Lebwohl et al., 2018). Eventually it leads to villous atrophy. Salem et al. studied the association between villous atrophy and ulcerative colitis, one of the conditions of IBD, long back in 1964 (Salem et al., 1964).

In May 2020, Pinto-sanchez et al. conducted a systematic review and meta-analysis to study the correlation of IBD with CD. They assessed the risk of CD in IBD patients and the risk of IBD in CD patients based on prognosis and prevalence studies in adult and/or pediatric populations from various geographical locations using healthcare and bioinformatic databases accessed till June 25, 2019 (Pinto-Sanchez et al., 2020). The outcomes of this research confirm and extend the investigation of CD and IBD association in adult patients conducted by Oxford et al. (2013) and Shah et al. (2019). On the one hand, the review demonstrated nine times increased risk of IBD in CD patients, greater risk of Crohn's disease as compared to ulcerative colitis. On the other hand, IBD patients are also likely to catch $\mathrm{CD}$ as well, however the chances are less than $\mathrm{CD}$ patients getting diagnosed with IBD (PintoSanchez et al., 2020).

\section{Conclusion and Future Perspective}

The ever-increasing numbers of cases for the aforementioned autoimmune diseases around the world shows a clear loophole in the basic diet that we consume. Celiac disease has a correlation with these diseases but further research needs to be conducted to establish a direct association of celiac disease and the autoimmune diseases. The more important question for us to ask is, whether a switch in our dietary habits or a reduced intake in the quantity of gluten will be just sufficient to overcome $\mathrm{CD}$ and will it have any impact on the associated diseases? We don't know the answers yet but further research in the field will open up new avenues for the world to explore human health and the role of a balanced diet and the impact of nutrition on our overall health.

\section{Abbreviations}

ASD: Autism Spectrum Disorder

BB rat: Bio breeding rat

BBDP rat: Bio breeding Diabetes Prone rat

BMI: Body Mass Index

CD: Celiac Disease

CNS: Central Nervous System

ENS: Enteric Nervous System

GADA: Glutamic acid decarboxylase autoantibodies

GFD: Gluten-free diet

GI: Gastrointestinal

GALT: Gut Associated Lymphoid Tissue

IA-2A: tyrosine islet antigen-2 autoantibodies

IgA: Immunoglobulin A

IgG: Immunoglobulin G

MRI: Magnetic Resonance Imaging

MS: Multiple Sclerosis

NOD mice: Non-Obese Diabetic mice

tTG: Tissue Transglutaminase

T1D: Type 1 Diabetes

TEER: Transepithelial electrical resistance

\section{Conflict of interest}

There are no conflicts to declare.

\section{Acknowledgement}

This work was supported by Dr. Jitendra Kumar from Bangalore Bio-innovation Centre and Dr. Balachandran from Vellore Institute of Technology-TBI. This is not a funded review work. Piyush Bhanu designed the paper. Sakshi Buchke, Bhavika Mehta, Maitrali Relekar and Anusuiya Bora prepared the manuscript. Dr. Piyush Varsha and Dr. Jitendra Kumar critically reviewed the manuscript.

\section{References}

[1] Akobeng A, Ramanan A, Basude D. EFFECT OF BREAST-FEEDING ON RISK OF COELIAC DISEASE: A SYSTEMATIC REVIEW AND METAANALYSIS OF OBSERVATIONAL STUDIES: PG118. Journal of Pediatric Gastroenterology and Nutrition. 2005;40:638-9.

[2] Awika JM. Major cereal grains production and use around the world. Advances in cereal science: implications to food processing and health promotion: ACS Publications; 2011;1-13.

[3] Bai D, Yip BHK, Windham GC, et al. Association of genetic and environmental factors with autism in a 5country cohort. JAMA psychiatry. 2019;76:1035-43.

[4] Bao F, Yu L, Babu S, et al. One third of HLA DQ2 homozygous patients with type 1 diabetes express celiac disease-associated transglutaminase autoantibodies. Journal of autoimmunity. 1999;13:143-8.

[5] Barcia G, Posar A, Santucci M, et al. Autism and coeliac disease. Journal of autism and developmental disorders. 2008;38:407.

[6] Batur-Caglayan H, Irkec C, Yildirim-Capraz I, et al. A case of multiple sclerosis and celiac disease. Case reports in neurological medicine. 2013;2013.

[7] Bosi E, Molteni L, Radaelli M, et al. Increased intestinal permeability precedes clinical onset of type 1 diabetes. Diabetologia. 2006;49:2824-7.

[8] Bruun SW, Josefsen K, Tanassi JT, et al. Large gliadin peptides detected in the pancreas of NOD and healthy mice following oral administration. Journal of diabetes research. 2016;2016.

[9] Bulger K, Griffin M, Dervan P, et al. Coeliac disease in association with inflammatory bowel disease. Postgraduate medical journal. 1988;64:336.

[10] Caio G, Volta U, Sapone A, et al. Celiac disease: a comprehensive current review. BMC medicine. 2019;17:1-20.

[11] Calderoni S, Santocchi E, Del Bianco T, et al. Serological screening for Celiac Disease in 382 preschoolers with Autism Spectrum Disorder. Italian journal of pediatrics. 2016;42:1-6.

[12] Camara-Lemarroy CR, Metz L, Meddings JB, et al. The intestinal barrier in multiple sclerosis: implications for pathophysiology and therapeutics. Brain. 2018;141:190016.

[13] Camarca ME, Mozzillo E, Nugnes R, et al. Celiac disease in type 1 diabetes mellitus. Italian journal of pediatrics. 2012;38:1-7.

[14] Camilleri á, Madsen K, Spiller R, et al. Intestinal barrier function in health and gastrointestinal disease. Neurogastroenterology \& Motility. 2012;24:503-12. 
[15] Caminero A, Meisel M, Jabri B, et al. Mechanisms by which gut microorganisms influence food sensitivities. Nature Reviews Gastroenterology \& Hepatology. 2019;16:7-18.

[16] Casella G, D'Incà R, Oliva L, et al. Prevalence of celiac disease in inflammatory bowel diseases: An IG-IBD multicentre study. Digestive and liver disease. 2010;42:175-8.

[17] Cermak SA, Curtin C, Bandini LG. Food selectivity and sensory sensitivity in children with autism spectrum disorders. Journal of the American Dietetic Association. 2010;110:238-46.

[18] Chaidez V, Hansen RL, Hertz-Picciotto I. Gastrointestinal problems in children with autism, developmental delays or typical development. Journal of autism and developmental disorders. 2014;44:1117-27.

[19] Chirdo F, Rumbo M, Anon M, et al. Presence of high levels of non-degraded gliadin in breast milk from healthy mothers. Scandinavian journal of gastroenterology. 1998;33:1186-92.

[20] Cottone $\mathrm{M}$, Marrone $\mathrm{C}$, Casà $\mathrm{A}$, et al. Familial occurrence of inflammatory bowel disease in celiac disease. Inflammatory bowel diseases. 2003;9:321-3.

[21] Dall M, Calloe K, Haupt-Jorgensen M, et al. Gliadin Fragments and a Specific Gliadin 33-mer Peptide Close K ATP Channels and Induce Insulin Secretion in INS-1E Cells and Rat Islets of Langerhans. PloS one. 2013;8:e66474.

[22] De Goffau MC, Luopajärvi K, Knip $M$, et al. Fecal microbiota composition differs between children with $\beta$ cell autoimmunity and those without. Diabetes. 2013;62:1238-44.

[23] De Re V, Caggiari L, Tabuso M, et al. The versatile role of gliadin peptides in celiac disease. Clinical biochemistry. 2013;46:552-60.

[24] Di Liberto D, D'Anneo A, Carlisi D, et al. Brain opioid activity and oxidative injury: Different molecular scenarios connecting celiac disease and autistic spectrum disorder. Brain Sciences. 2020;10:437.

[25] EL-ALAMEEY IR, AHMED HH, EID IM, et al. SERUM ANTIGLIADIN ANTIBODIES IN EGYPTIAN CHILDREN WITH AUTISM SPECTRUM DISORDER: RELATIONSHIP TO GASTROINTESTINAL SYMPTOMS, BEHAVIORAL AND SOCIAL COMMUNICATIONS. inflammation. 2018;3:4.

[26] Fallang L-E, Roh S, Holm A, et al. Complexes of two cohorts of CLIP peptides and HLA-DQ2 of the autoimmune DR3-DQ2 haplotype are poor substrates for HLA-DM. The Journal of Immunology. 2008;181:545161.

[27] Faras H, Al Ateeqi N, Tidmarsh L. Autism spectrum disorders. Annals of Saudi medicine. 2010;30:295-300.

[28] Fasano A. Zonulin and its regulation of intestinal barrier function: the biological door to inflammation, autoimmunity, and cancer. Physiological reviews. 2011.

[29] Festen EA, Goyette P, Green T, et al. A meta-analysis of genome-wide association scans identifies IL18RAP, PTPN2, TAGAP, and PUS10 as shared risk loci for Crohn's disease and celiac disease. PLoS Genet. 2011;7:e1001283.

[30] Fiorentino M, Sapone A, Senger S, et al. Blood-brain barrier and intestinal epithelial barrier alterations in autism spectrum disorders. Molecular autism. 2016;7:117.

[31] Fouda GG, Martinez DR, Swamy GK, et al. The Impact of IgG transplacental transfer on early life immunity. Immunohorizons. 2018;2:14-25.

[32] Funda DP, Kaas A, Bock T, et al. Gluten-free diet prevents diabetes in NOD mice. Diabetes/metabolism research and reviews. 1999;15:323-7.

[33] Galli-Tsinopoulou A, Nousia-Arvanitakis S, Dracoulacos $\mathrm{D}$, et al. Autoantibodies predicting diabetes mellitus type I in celiac disease. Hormone Research in Paediatrics. 1999;52:119-24.

[34] Garcia-Bennett AE, Ballell L. Non-absorbable mesoporous silica for the development of protein sequestration therapies. Biochemical and biophysical research communications. 2015;468:428-34.

[35] Gardner RM, Samuelsson I, Severance EG, et al. Maternal antibodies to gliadin and autism spectrum disorders in offspring-A population-based case-control study in Sweden. bioRxiv. 2020.

[36] Gillberg R, Dotevall G, Åhrén C. Chronic inflammatory bowel disease in patients with coeliac disease. Scandinavian journal of gastroenterology. 1982;17:4916.

[37] Giongo A, Gano KA, Crabb DB, et al. Toward defining the autoimmune microbiome for type 1 diabetes. The ISME journal. 2011;5:82-91.

[38] Haider MN. Dynamic Cerebral Blood Flow Regulation in Adolescent Athletes with Sports-Related Concussion: State University of New York at Buffalo; 2021.

[39] Harris KG, Chang EB. The intestinal microbiota in the pathogenesis of inflammatory bowel diseases: new insights into complex disease. Clinical Science. 2018;132:2013-28.

[40] Hisamatsu T, Erben U, Kühl AA. The role of t-cell subsets in chronic inflammation in celiac disease and inflammatory bowel disease patients: More common mechanisms or more differences? Inflammatory intestinal diseases. 2016;1:52-62.

[41] Hmida NB, Ahmed MB, Moussa A, et al. Impaired control of effector $\mathrm{T}$ cells by regulatory $\mathrm{T}$ cells: a clue to loss of oral tolerance and autoimmunity in celiac disease? American Journal of Gastroenterology. 2012;107:604-11.

[42] Hollander D, Kaunitz JD. The "Leaky Gut": tight junctions but loose associations? : Springer; 2020.

[43] Holmes G. Screening for coeliac disease in type 1 diabetes. Archives of Disease in Childhood. 2002;87:495-8.

[44] Jabri B, Abadie V. IL-15 functions as a danger signal to regulate tissue-resident $\mathrm{T}$ cells and tissue destruction. Nature Reviews Immunology. 2015;15:771-83.

[45] Jabri B, Sollid LM. T cells in celiac disease. The Journal of Immunology. 2017;198(8):3005-14.

[46] Jensen PE. Recent advances in antigen processing and presentation. Nature immunology. 2007;8:1041-8.

[47] Kamboj AK, Oxentenko AS. Clinical and histologic mimickers of celiac disease. Clinical and translational gastroenterology. 2017;8:e114.

[48] Kitis G, Holmes G, Cooper B, et al. Association of coeliac disease and inflammatory bowel disease. Gut. 1980;21:636-41. 
[49] Knudsen KB, Hansen I. Gastrointestinal implications in pigs of wheat and oat fractions: 1. Digestibility and bulking properties of polysaccharides and other major constituents. British journal of nutrition. 1991;65:217-32.

[50] Kumar P, O'Donoghue D, Gibson J, et al. The existence of inflammatory bowel lesions in gluten-sensitive enteropathy. Postgraduate medical journal. 1979;55:7536.

[51] Lahiri DK, Sokol DK, Erickson C, et al. Autism as early neurodevelopmental disorder: evidence for an sAPP $\alpha$ mediated anabolic pathway. Frontiers in cellular neuroscience. 2013;7:94.

[52] Lammers KM, Lu R, Brownley J, et al. Gliadin induces an increase in intestinal permeability and zonulin release by binding to the chemokine receptor CXCR3. Gastroenterology. 2008;135:194-204. e3.

[53] Lawlor G, Peppercorn MA. New genetic data support an association between celiac disease and inflammatory bowel disease. Inflammatory bowel diseases. 2011;17:E80-E1.

[54] Lebwohl B, Sanders DS, Green PH. Coeliac disease. The Lancet. 2018;391:70-81.

[55] Leeds JS, Höroldt BS, Sidhu R, et al. Is there an association between coeliac disease and inflammatory bowel diseases? A study of relative prevalence in comparison with population controls. Scandinavian journal of gastroenterology. 2007;42:1214-20.

[56] Lombardi F, Franzese A, Iafusco D, et al. Bone involvement in clusters of autoimmune diseases: just a complication? Bone. 2010;46:551-5.

[57] Ludvigsson JF, Reichenberg A, Hultman CM, et al. A nationwide study of the association between celiac disease and the risk of autistic spectrum disorders. JAMA psychiatry. 2013;70:1224-30.

[58] Lundin KE, Wijmenga C. Coeliac disease and autoimmune disease-genetic overlap and screening. Nature reviews Gastroenterology \& hepatology. 2015;12:507.

[59] Malik TA. Inflammatory bowel disease: historical perspective, epidemiology, and risk factors. Surgical Clinics. 2015;95:1105-22.

[60] Marietta EV, Gomez AM, Yeoman C, et al. Low incidence of spontaneous type 1 diabetes in non-obese diabetic mice raised on gluten-free diets is associated with changes in the intestinal microbiome. PloS one. 2013;8:e78687.

[61] McElhanon BO, McCracken C, Karpen S, et al. Gastrointestinal symptoms in autism spectrum disorder: a meta-analysis. Pediatrics. 2014;133:872-83.

[62] Mehanna M, Mneimneh A. Updated but not outdated "Gliadin": A plant protein in advanced pharmaceutical nanotechnologies. International Journal of Pharmaceutics. 2020:119672.

[63] Meisel M, Mayassi T, Fehlner-Peach H, Koval JC, et al. Interleukin-15 promotes intestinal dysbiosis with butyrate deficiency associated with increased susceptibility to colitis. The ISME journal. 2017;11:1530.

[64] Mocan O, Dumitraşcu DL. The broad spectrum of celiac disease and gluten sensitive enteropathy. Clujul Medical. 2016;89:335.

[65] Mokarizadeh A, Esmaeili P, Soraya H, et al. Antibody against $\alpha$-gliadin 33-mer peptide: Is the key initiating factor for development of multiple sclerosis during gluten sensitivity? Journal of Medical Hypotheses and Ideas. 2015;9:38-44.

[66] Murri M, Leiva I, Gomez-Zumaquero JM, et al. Gut microbiota in children with type 1 diabetes differs from that in healthy children: a case-control study. BMC medicine. 2013;11:1-12.

[67] Norris JM, Barriga K, Hoffenberg EJ, et al. Risk of celiac disease autoimmunity and timing of gluten introduction in the diet of infants at increased risk of disease. Jama. 2005;293:2343-51.

[68] Norris JM, Barriga K, Klingensmith G, et al. Timing of initial cereal exposure in infancy and risk of islet autoimmunity. Jama. 2003;290:1713-20.

[69] Ordonez F, Lacaille F, Canioni D, et al. Pediatric ulcerative colitis associated with autoimmune diseases: a distinct form of inflammatory bowel disease? Inflammatory bowel diseases. 2012;18:1809-17.

[70] Oxford EC, Nguyen DD, Sauk J, et al. Impact of coexistent celiac disease on phenotype and natural history of inflammatory bowel diseases. The American journal of gastroenterology. 2013;108.

[71] Pascual V, Dieli-Crimi R, López-Palacios N, et al. Inflammatory bowel disease and celiac disease: overlaps and differences. World journal of gastroenterology: WJG. 2014;20:4846.

[72] Patel J, Agasti A, Rao S, et al. Celiac disease preceding Crohn's disease? Tropical Gastroenterology. 2011;32:236-8.

[73] Pilli D, Zou A, Tea F, et al. Expanding role of T cells in human autoimmune diseases of the central nervous system. Frontiers in immunology. 2017;8:652.

[74] Pinto-Sanchez MI, Seiler CL, Santesso N, et al. Association between inflammatory bowel diseases and celiac disease: a systematic review and meta-analysis. Gastroenterology. 2020;159:884-903. e31.

[75] Pocecco M, Ventura A. Coeliac disease and insulindependent diabetes mellitus: a causal association? Acta Paediatrica. 1995;84:1432-3.

[76] Pociot F, McDermott M. Genetics of type 1 diabetes mellitus. Genes \& Immunity. 2002;3:235-49.

[77] Pos W, Sethi DK, Call MJ, et al. Crystal structure of the HLA-DM-HLA-DR1 complex defines mechanisms for rapid peptide selection. Cell. 2012;151:1557-68.

[78] Poulain C, Johanet C, Delcroix C, et al. Prevalence and clinical features of celiac disease in 950 children with type 1 diabetes in France. Diabetes \& metabolism. 2007;33:453-8.

[79] Quan J, Panaccione N, King J, et al. A257 ASSOCIATION BETWEEN CELIAC DISEASE AND AUTISM SPECTRUM DISORDER: A SYSTEMATIC REVIEW. Journal of the Canadian Association of Gastroenterology. 2019;2:502-3.

[80] Rao M, Gershon MD. The bowel and beyond: the enteric nervous system in neurological disorders. Nature reviews Gastroenterology \& hepatology. 2016;13:517.

[81] Reichelt KL, Jensen D. IgA antibodies against gliadin and gluten in multiple sclerosis. Acta neurologica scandinavica. 2004;110:239-41.

[82] Rodrigo L, Hernández-Lahoz C, Fuentes D, et al. Prevalence of celiac disease in multiple sclerosis. BMC neurology. 2011;11:1-7. 
[83] Rubio-Tapia A, Hill ID, Kelly CP, et al. American College of Gastroenterology clinical guideline: diagnosis and management of celiac disease. The American journal of gastroenterology. 2013;108:656.

[84] Salem S, Truelove S, Richards W. Small-intestinal and gastric changes in ulcerative colitis: a biopsy study. British medical journal. 1964;1:394.

[85] Santiago JL, Martínez A, Núñez C, et al. Association of MYO9B haplotype with type 1 diabetes. Human immunology. 2008;69:112-5.

[86] Sapone A, De Magistris L, Pietzak M, et al. Zonulin upregulation is associated with increased gut permeability in subjects with type 1 diabetes and their relatives. Diabetes. 2006;55:1443-9.

[87] Sblattero D, Maurano F, Mazzarella G, et al. Characterization of the anti-tissue transglutaminase antibody response in nonobese diabetic mice. The Journal of Immunology. 2005;174:5830-6.

[88] Schedel J, Rockmann F, Bongartz T, et al. Association of Crohn's disease and latent celiac disease: a case report and review of the literature. International journal of colorectal disease. 2005;20:376-80.

[89] Schneeberger EE, Lynch RD. The tight junction: a multifunctional complex. American Journal of Physiology-Cell Physiology. 2004;286:C1213-C28.

[90] Schneider S, Wright CM, Heuckeroth RO. Unexpected roles for the second brain: enteric nervous system as master regulator of bowel function. Annual review of physiology. 2019;81:235-59.

[91] Scott FW, Marliss EB. Conference summary: diet as an environmental factor in development of insulindependent diabetes mellitus. Canadian journal of physiology and pharmacology. 1991;69:311-9.

[92] Shah A, Walker M, Burger D, et al. Link between celiac disease and inflammatory bowel disease. Journal of clinical gastroenterology. 2019;53:514-22.

[93] Shaygannejad V, Ghasemi M, Mirmohamadsadeghi M. Multiple sclerosis or neurological manifestations of Celiac disease. Advanced biomedical research. 2013;2.

[94] Shewry P. What is gluten-Why is it special? Frontiers in nutrition. 2019;6:101.

[95] Shewry PR, Halford NG, Belton PS, et al. The structure and properties of gluten: an elastic protein from wheat grain. Philosophical Transactions of the Royal Society of London Series B: Biological Sciences. 2002;357:133-42.

[96] Shimada S, Tanigawa T, Watanabe T, et al. Involvement of gliadin, a component of wheat gluten, in increased intestinal permeability leading to non-steroidal antiinflammatory drug-induced small-intestinal damage. PloS one. 2019;14:e0211436.

[97] Slifka MK, Amanna IJ. Passive immunization. Plotkin's Vaccines. 2018:84.

[98] Soukou S, Brockmann L, Bedke T, et al. Role of IL-10 receptor signaling in the function of CD4+ T-regulatory type 1 cells: T-cell therapy in patients with inflammatory bowel disease. Critical reviews ${ }^{\mathrm{TM}}$ in immunology. 2018;38.

[99] Srinivasan B, Kolli AR, Esch MB, et al. TEER measurement techniques for in vitro barrier model systems. Journal of laboratory automation. 2015;20:10726.

[100] Stene LC, Oikarinen S, Hyöty H, et al. Enterovirus infection and progression from islet autoimmunity to type 1 diabetes: the Diabetes and Autoimmunity Study in the Young (DAISY). Diabetes. 2010;59:3174-80.

[101] Stepniak D, Spaenij-Dekking L, Mitea C, et al. Highly efficient gluten degradation with a newly identified prolyl endoprotease: implications for celiac disease. American Journal of Physiology-Gastrointestinal and Liver Physiology. 2006;291:G621-G9.

[102] Valerio G, Maiuri L, Troncone R, et al. Severe clinical onset of diabetes and increased prevalence of other autoimmune diseases in children with coeliac disease diagnosed before diabetes mellitus. Diabetologia. 2002;45:1719-22.

[103] Ventura A, Neri E, Ughi C, et al. Gluten-dependent diabetes-related and thyroid-related autoantibodies in patients with celiac disease. The Journal of pediatrics. 2000;137:263-5.

[104] Virtanen SM, Knip M. Nutritional risk predictors of $\beta$ cell autoimmunity and type 1 diabetes at a young age. The American journal of clinical nutrition. 2003;78:1053-67.

[105] Vojdani A, O'Bryan T, Green J, et al. Immune response to dietary proteins, gliadin and cerebellar peptides in children with autism. Nutritional neuroscience. 2004;7:151-61.

[106] Vojdani A, Pangborn J, Vojdani E, et al. Infections, toxic chemicals and dietary peptides binding to lymphocyte receptors and tissue enzymes are major instigators of autoimmunity in autism. International journal of immunopathology and pharmacology. 2003;16:189-99.

[107] Vojdani A, Vojdani E. Gluten and non-gluten proteins of wheat as target antigens in autism, Crohn's and celiac disease. Journal of Cereal Science. 2017;75:252-60.

[108] Volta U, Tovoli F, Caio G. Clinical and immunological features of celiac disease in patients with type 1 diabetes mellitus. Expert review of gastroenterology \& hepatology. 2011;5:479-87.

[109] Watts T, Berti I, Sapone A, et al. Role of the intestinal tight junction modulator zonulin in the pathogenesis of type I diabetes in BB diabetic-prone rats. Proceedings of the National Academy of Sciences. 2005;102:2916-21.

[110] Wrigley C, Békés F, Bushuk W. Gluten: A balance of gliadin and glutenin. Gliadin and glutenin: The unique balance of wheat quality. 2006:3-32.

[111] Yang A, Chen Y, Scherl E, et al. Inflammatory bowel disease in patients with celiac disease. Inflammatory bowel diseases. 2005;11:528-32.

[112] Yang J, Chow I-T, Sosinowski T, et al. Autoreactive T cells specific for insulin $\mathrm{B}$ : 11-23 recognize a lowaffinity peptide register in human subjects with autoimmune diabetes. Proceedings of the National Academy of Sciences. 2014;111:14840-5.

[113] Yeung W-CG, Rawlinson WD, Craig ME. Enterovirus infection and type 1 diabetes mellitus: systematic review and meta-analysis of observational molecular studies. Bmj. 2011;342.

[114] Zhang X-M, Liu C-Y, Shao Z-H. Advances in the role of helper $\mathrm{T}$ cells in autoimmune diseases. Chinese medical journal. 2020;133:968.

[115] Zhou Z, Reyes-Vargas E, Escobar H, et al. Type 1 diabetes associated HLA-DQ2 and DQ8 molecules are relatively resistant to HLA-DM mediated release of invariant chain-derived CLIP peptides. European journal of immunology. 2016;46:834-45. 
[116] Ziegler A-G, Schmid S, Huber D, et al. Early infant feeding and risk of developing type 1 diabetesassociated autoantibodies. Jama. 2003;290:1721-8.

Open Access This article is licensed under a Creative Commons Attribution 4.0 International License, which permits use, sharing, adaptation, distribution and reproduction in any medium or format, as long as you give appropriate credit to the original author(s) and the source, provide a link to the Creative Commons license, and indicate if changes were made. The images or other third party material in this article are included in the article's Creative Commons license, unless indicated otherwise in a credit line to the material. If material is not included in the article's Creative Commons license and your intended use is not permitted by statutory regulation or exceeds the permitted use, you will need to obtain permission directly from the copyright holder. To view a copy of this license, visit https://creativecommons.org/licenses/by/4.0/.

(C) The Author(s) 2021 